\title{
Hierarchical control of unmanned ground vehicle formations using multi-body approach
}

\author{
Béla Lantos, György Max
}

Budapest University of Technology and Economics, Hungary

H-1117 Budapest, Magyar Tudósok krt. 2., Hungary

E-mail:lantos@iit.bme.hu,max@iit.bme.hu

\begin{abstract}
The paper deals with the formation control of Unmanned Ground Vehicles (UGVs) moving in horizontal plane. The control system consists of the high level centralized formation control of the UGVs and the low level decentralized PID type suspension, speed and steering control of the different vehicles. Both problems are discussed in multi-body assumptions. The paper presents the generalization of the multi-body method for underactuated car-like vehicles, developed originally for fully-actuated surface ships. In order to simplify the design and implementation on the formation level, an approximate single track dynamic model was assumed for each vehicle. At low level a more realistic two track dynamic model is used in the form of a multibody system in tree structure. This realistic nonlinear model is obtained by using Appell's method, Pacejka's magic formula for tyre-road connections and kinematic constraints expressing the nullity of vertical accelerations of the contact points. The interface between the higher and lower control levels is presented in the form of acceleration and steering angle prescriptions (output of high level). The decentralized control system of each vehicle converts the specifications in smooth reference signals and performs the desired motion. Simulation results of the high level control of UGV formations are presented for sine-shaped and circular paths.
\end{abstract}

Keywords: Formation Control, Unmanned Ground Vehicles, Multi-Body Approach, Tree Structured Vehicle, Pacejka's Magic Formula, Contact Point Constraints, Robust PID Control

\section{Introduction}

Formation control design and implementation is a complex and time-critical problem for which a hierarchical control system will be suggested. The high level subsystem deals with the formation control of vehicles satisfying connection constraints equivalent to the formation. The problem is a multi-body one in the sense that many vehicles take part in the formation. In order to simplify the design and realization at the formation level, approximate single track dynamic model will be assumed here for each vehicle. However, the realization needs a more realistic model for 
the vehicles, hence a two track model will be considered for each vehicle at the lower control level. The interface between the higher and the lower control levels will be presented in the form of acceleration and steering angle prescriptions for the different vehicles and produced as output of the formation control. At low level, the decentralized control system of each vehicle converts the specifications in sufficiently smooth reference signals and performs the desired control based on robust PID type suspension, speed and steering control. At this level each vehicle is considered as a real multi-body system in tree structure. The motion of the formation is the result of both levels.

For stabilization of ground vehicles (robots) in formation the fusion of potential field method, passivity theory, dynamic inversion and LMI technique is a theoretically well founded approach if the inertia of the car-like vehicles has to be taken into consideration [1], [2]. Synchronized path following based on the fusion of backstepping control and passivity theory was suggested for surface ships [3]. Unfortunately this method cannot be used for UGVs because the dynamic model of the vehicles does not satisfy the strict-feedback form which is assumed for backstepping control. Another approach may be multi-body interpretation of the formation resulting in constrained control. This method was successfully applied in the formation control of full-actuated surface ships [4]. For formation flight control of constraint multi-body system [5] presents an approach where the aircraft model is of point-mass type and only position distance constraints are considered. Ground vehicles are underactuated and in many cases their inertia cannot be neglected, thus the original formulation for ships has to be generalized. One aim of the paper is to elaborate the necessary modification of the theory and illustrate its applicability for car-like UGVs.

In general, the ground vehicle can be regarded as a multi-body system whose base is the mobile chassis and the wheels are the end effectors. Several methods are available to find the kinematics and dynamic models of mobile robots [6, 7], but they mostly build on simplifying kinematic constraints that do not take into account the three-dimensional forces between the wheel and the ground. Other recent works $[8,9]$ use robotic description for modeling and validation of cars, but they do not deal with closed loop control and do not take into account the lateral and longitudinal offset in the vehicle's centre of gravity point (CoG). Numerical methods and symbolic software (Symoro+, OpenSYMORO) are available to find the dynamic model based on Newton-Euler method [10]. Another often used method is the Lagrange technique. In this paper, an alternative approach is introduced that uses the concept of acceleration energy and eliminates a large number of numerical steps of the Newton-Euler method. The algorithm is based on Appell's method which directly computes the dynamic model of the composite system. Although using symbolic software these methods result in equivalent dynamic models, however for only numerical computations (without the use of the symbolic results) they have different computation time. Another aim of the work is to develop a complex vehicle control system that is capable of eliminating the rolling and pitching effect through active suspension control system as well as maintaining a prescribed velocity and steering angle profile in closed loop. Such a system helps also studying the interaction of the system with the environment. 
The structure of the paper is as follows. Section 2 summarizes the concept of the multi-body implementation for full-actuated systems (surface ships, robots etc.). Section 3 discusses the conversion of formation specifications into multi-body constraints. Section 4 gives the approximate dynamic model of a single ground vehicle and the generalization of the multi-body approach for UGVs. The simplified model consideres the tyre-road connections through the cornering stiffnesses which is a linear approximation. Section 5 presents the geometric and kinematic model and the tree structured topology of a single vehicle using the modified Denavit-Hartenberg form [11]. Here will be developed the vehicle's realistic two-track dynamic model using Appell's method by calculating the Gibbs functions of each segment. This section describes also the kinematic constraints and the external forces acting on the vehicle. The realistic model considers the tyre-road connections in the form of Pace$\mathrm{jka}$ 's magic formulas hence the model is nonlinear in the state variables. In Section 6 the decentralized low-level control system will be presented in short form containing the reference signal design and the concept of PID type active suspension, driving and steering control based on the realistic nonlinear vehicle model. Section 7 shows the simulation results for formation control of UGVs using multi-body approach. Finally Section 8 summarizes the conclusions and the main directions of future research.

\section{Fully actuated control of constrained multi-body sys- tems}

A single full-actuated marine vehicle moving in the horizontal plane can be modelled by

$$
\begin{gathered}
\dot{\eta}=R(\psi) v \\
M \dot{v}+n(v, \dot{v}, \eta)=\tau
\end{gathered}
$$

where $R(\psi)$ is the rotation matrix from body to the (quasi) inertia frame, $\eta=(x, y, \psi)^{T}$ is the position and orientation, $v=(u, v, r)^{T}$ is the linear and angular velocity, $M$ denotes the system inertia (for ships the rigid body inertia and the added mass) and $n$ contains the centripetal, Coriolis, damping and gravity effects. Notice the similarity to robot control in 6-DOF where $H(q) \ddot{q}+h(q, \dot{q})=\tau$ is the dynamic model of the robot in joint coordinates and the Jacobian $J(q)$ plays the role of the rotation matrix according to $\dot{x}=J(q) \dot{q}$.

If a set of constraints is given in the form of $C(\eta)=0 \in R^{p}$ in the inertia system and the constraints Jacobian is denoted by $W(\eta)=\frac{\partial C(\eta)}{\partial \eta}$ then, by using the results of [12], the motion equation is modified to

$M \dot{v}+n(\nu, \dot{v}, \eta)=\tau+\tau_{c}$

where the constraint force $\tau_{c}$ has the form $\tau_{c}=-W(\eta)^{T} \lambda$ and $\lambda$ is the Lagrange multiplier. 
Transforming the motion equation into the inertia frame and using the fact that $R^{T} \tau_{\eta}=\tau \Rightarrow \tau_{\eta}=R \tau$, it yields

$M_{\eta}(\eta) \ddot{\eta}+n_{\eta}(\nu, \dot{v}, \eta)=\tau_{\eta}-R(\psi) W(\eta)^{T} \lambda$.

It follows from $C(\eta)=0$ that

$\dot{C}(\eta)=\frac{\partial C}{\partial \eta} \dot{\eta}=W(\eta) \dot{\eta}=0, \quad \ddot{C}(\eta)=W(\eta) \ddot{\eta}+\dot{W}(\eta) \dot{\eta}=0$.

Adding stabilizing terms we choose

$\ddot{C}=-K_{d} \dot{C}-K_{p} C$

with $K_{d}, K_{p}$ diagonal and having positive elements. Then $s^{2}+k_{d i} s+k_{p i}=s^{2}+$ $2 \xi \omega_{0} s+\omega_{0}^{2}=0$ is stable if $k_{d i}=2 \xi \omega_{0}$ and $k_{p i}=\omega_{0}^{2}$ where $\xi>0$ is the damping and $\omega_{0}$ is the undamped eigenfrequency. In this case it follows

$W M_{\eta}^{-1}\left(\tau_{\eta}-n_{\eta}-R W^{T} \lambda\right)+\dot{W} \dot{\eta}=-K_{d} \dot{C}-K_{p} C$

$\lambda=\left(W M_{\eta}^{-1} R W^{T}\right)^{-1}\left[W M_{\eta}^{-1}\left(\tau_{\eta}-n_{\eta}\right)+\dot{W} \dot{\eta}+K_{d} \dot{C}+K_{p} C\right]$

if $W M_{\eta}^{-1} R W^{T}$ is invertible which is satisfied if $W$ has full row rank.

If there are $n$ vehicles then we can collect vectors into new vectors and matrices into new blockdiagonal matrices. The resulting vectors and matrices will be denoted further on by $\eta, n_{\eta}, \tau_{\eta}, \tau_{c}$ and $M_{\eta}$, respectively. The prescribed formation can be converted to the constraint $C(\eta)=0$ having Jacobian $W(\eta)$.

The constraint force for the $i$ th vehicle is

$$
\begin{aligned}
\tau_{c i}=\sum_{k \in A_{c}^{i}} \sum_{j \in B_{k}}- & W_{k i}^{T}\left(W_{k} M_{\eta, i j}^{-1} R_{i j}^{-1 T} W_{k}^{T}\right)^{-1} \times \\
& {\left[W_{k i} M_{\eta, i j}\left(\tau_{\eta, i j}-n_{\eta, i j}\right)+K_{d, k i} \dot{C}_{k i}+K_{p, k i} C_{k i}\right] }
\end{aligned}
$$

where $A_{c}^{i}$ is the index set of vehicles staying in connection with vehicle $i, B_{k}$ is the index of constraints selected by index $k$ and $W_{k i}=0$ for $k \notin A_{c}^{i}$.

\section{Conversion of formation specifications to multi-body constraints}

In the sequel the indexes $p, o$ and $d$ denote position, orientation and desired value, respectively, furthermore $f$ is for fixed, $t t$ for time dependent value and $r$ for relative value between two vehicles. For simplicity denote here $\xi_{i}=\left(x_{i}, y_{i}\right)^{T}$ the position and $\psi_{i}$ the orientation of vehicle $i$, and let their collected vectors be $\xi$ and $\psi$, respectively.

Desired position and orientation constraints. The position of vehicle $i$ is forced to $\xi_{d}$ by the constraint

$C_{p}(\xi)=\xi_{i}-\xi_{d}=0$. 
For at least three times differentiable desired path $\xi_{d}(t)$ we have

$C_{t t}(\xi)=\xi_{i}-\xi_{d}(t)=0$.

Similarly, for orientation constraints it yields

$C_{o}(\psi)=\psi_{i}-\psi_{d}=0, \quad C_{o, t t}(\psi)=\psi_{i}-\psi_{d}(t)=0$.

Distance constraints. If the distance $r_{i j}$ should be satisfied between vehicle $i$ and $j$ then the appropriate constraint is

$C_{r d}(\xi)=\left(\xi_{i}-\xi_{j}\right)^{T}\left(\xi_{i}-\xi_{j}\right)-r_{i j}^{2}=0$.

Fixed relative position and orientation constraints. For prescribed relative position and orientation between two vehicles the constraints are

$C_{f p}(\xi)=\xi_{i}-\xi_{j}-p_{i j}=0, \quad C_{f o}(\psi)=\psi_{i}-\psi_{j}-o_{i j}=0$.

Combined constraints. If for example $C_{r d}$ and $C_{p}$ are two constraints to which the constraint forces are $W_{r d}^{T} \lambda_{r d}$ and $W_{p}^{T} \lambda_{p}$ then they can be combined to

$W^{T} \lambda=\left[W_{r d}^{T} W_{p}^{T}\right]\left[\begin{array}{c}\lambda_{r d} \\ \lambda_{p}\end{array}\right]$

Formation topology. Typical formation specifications can be converted to a resulting constraint set by using the above steps and their combinations. We shall assume that redundant constraints have already been omitted and there are no contradictions amongst the constraints which means that the resulting $W$ has full row rank.

Master vehicle and followers. We can specify a master vehicle for which the desired path and path velocity will be designed. Specifications for the other vehicles can be derived from them if the formation type is chosen. Typical formations may be longitudinal, transversal, $\mathrm{V}$-shaped and circular ones.

If $x_{r}(t), y_{r}(t)$ is the desired reference path for the master vehicle then its desired reference orientation can be determined by $\psi_{r}(t)=\arctan 2\left(\dot{y}_{r}(t), \dot{x}_{r}(t)\right)$. Denoting the relative position of vehicle $i$ to the master vehicle by $p_{x_{i}}, p_{y_{i}}$ then the following constraints have to be introduced:

$C_{m, t t}(\eta)=\left[\begin{array}{c}x_{m}-x_{r}(t) \\ y_{m}-y_{r}(t) \\ \psi_{m}-\psi_{r}(t)\end{array}\right], \quad C_{i, t t}(\eta)=\left[\begin{array}{c}x_{i}-x_{r}(t)-p_{x_{i}}(t) \\ y_{i}-y_{r}(t)-p_{y_{i}}(t) \\ \psi_{i}-\psi_{r}(t)\end{array}\right] i \neq m$.

$C(\eta)=\left[\begin{array}{llll}C_{m, t t}^{T}(\eta) & \cdots & C_{i, t t}^{T}(\eta) & \cdots\end{array}\right]^{T}$.

If $W$ denotes the Jacobian of $C$ then it yields

$W^{T} \lambda=\left[\begin{array}{llll}W_{m, t t}^{T} & \cdots & W_{i, t t}^{T} & \cdots\end{array}\right]\left[\begin{array}{llll}\lambda_{m, t t}^{T} & \cdots & \lambda_{i, t t}^{T} & \cdots\end{array}\right]^{T}$.

Since in each row of $C$ appears only a single variable hence in case of the above convention $W=I$ and $\dot{W}=0$ which simplifies the computations. 


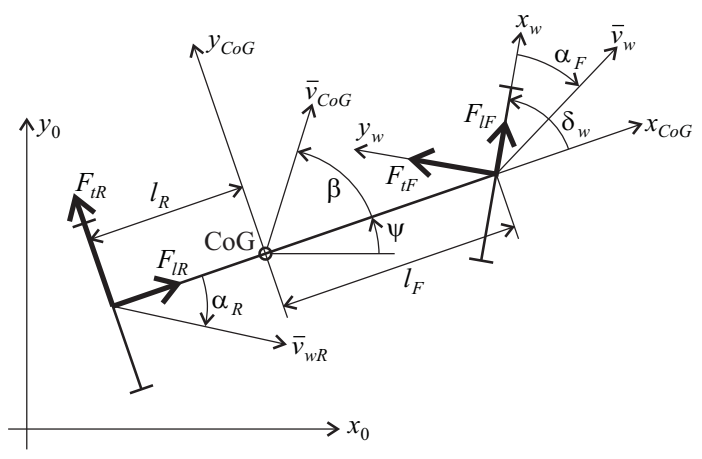

Figure 1

Simplified sketch of a single ground vehicle

\section{Multi-body approach for underactuated UGVs in for- mation}

The high level formation control design is a complex problem. Therefore, as usual in the literature, we apply the single-track vehicle model in order to make the computations easier and more efficient. The simplified sketch of a single car-like ground vehicle moving in horizontal plane is shown in Fig. 1.

\subsection{Simplified dynamic model of a single vehicle}

The dynamic model of a single ground vehicle can be written in the form

$$
\begin{array}{lll}
\dot{x}=v \cos (\phi) & \dot{\phi}=\frac{a_{11}}{v} \beta+\frac{a_{12}}{v^{2}} r+\frac{b_{1}}{v} \delta_{w} & \dot{r}=a_{21} \beta+\frac{a_{22}}{v} r+b_{2} \delta_{w} \\
\dot{y}=v \sin (\phi) & \dot{\beta}=\frac{a_{11}}{v} \beta+\left(\frac{a_{12}}{v^{2}}-1\right) r+\frac{b_{1}}{v} \delta_{w} & \dot{v}=\alpha
\end{array}
$$

where $q_{1}=(x, y)^{T}$ is the position, $v$ is the absolute value of the velocity, $\psi$ is the orintation, $\beta$ is the side slip angle, $\phi=\beta+\psi, \delta_{w}$ is the steering angle and $\alpha$ is the longitudinal acceleration, see [2]. Here we used the notations

$$
\begin{array}{lll}
a_{11}=-\frac{c_{F}+c_{R}}{m_{v}} & a_{12}=\frac{c_{R} l_{R}-c_{F} l_{F}}{m_{v}} & b_{1}=\frac{c_{F}}{m_{v}} \\
a_{21}=\frac{c_{R} l_{R}-c_{F} l_{F}}{I_{z}} & a_{22}=-\frac{c_{R} l_{R}^{2}+c_{F} l_{F}^{2}}{I_{z}} & b_{2}=\frac{c_{F} l_{F}}{I_{z}}
\end{array}
$$

where $m_{v}$ is the mass, $I_{z}$ is the inertia moment of the vehicle and $c_{F}, c_{R}$ are the cornering stiffnesses assumed to be constant.

For heavy-duty cars we assumed $a_{11}=-147.1481, a_{12}=0.0645, a_{21}=0.0123$, $a_{22}=-147.1494, b_{1}=66.2026$ and $b_{2}=31.9835$, all in standard SI units.

With $\bar{x}=(x, y, \phi, \beta, r, v)^{T}$ and $u=\left(\delta_{w}, \alpha\right)^{T}$ the system can be brought to the parameter dependent input affine form $\dot{\bar{x}}=\bar{A}(x, \rho)+\bar{B}(x, \rho) u$ where $\rho=\left(v, v^{2}\right)^{T}$ is the parameter vector. 


\subsection{Generalization of multi-body interpretation for UGVs}

Since each vehicle is underactuated and the number of input signals is two, we can prescribe only limited type of constraints. From physical consideration, the variables for which constraints can be specified, will be the position coordinates $x, y$. Hence, we omit orientation parts from the set of constraints. However, if the position follows its prescribed path and the side slip angle is zero (except for short transients), then the vehicle's velocity is parallel to the tangent of the path and its orientation is acceptable. For small side slip angle $\beta$ the assumption is fulfilled.

Choosing new state variables according to $q_{1}=(x, y)^{T}, q_{2}=(\dot{x}, \dot{y})^{T}$ and $q_{3}=(\beta, r)^{T}$, and applying the usual notation in robotics $C_{\phi}=\cos (\phi)$ and $S_{\phi}=\sin (\phi)$, then we obtain

$\dot{q}_{1}=\left[\begin{array}{l}v C_{\phi} \\ v S_{\phi}\end{array}\right]$

$\dot{q}_{2}=\left[\begin{array}{c}\dot{v} C_{\phi}-v S_{\phi} \dot{\phi} \\ \dot{v} S_{\phi}+v C_{\phi} \dot{\phi}\end{array}\right]=\left[\begin{array}{c}\alpha C_{\phi}-v S_{\phi}\left[\left(a_{11} / v\right) \beta+\left(a_{12} / v^{2}\right) r+\left(b_{1} / v\right) \delta_{w}\right] \\ \alpha S_{\phi}+v C_{\phi}\left[\left(a_{11} / v\right) \beta+\left(a_{12} / v^{2}\right) r+\left(b_{1} / v\right) \delta_{w}\right]\end{array}\right]$

from which follows $\ddot{q}_{1}=P(\phi) z+Q(\phi) \tau$, where $z=(\beta, r / v)^{T}, \tau=\left(\delta_{w}, \alpha\right)^{T}$ and

$P(\phi)=\left[\begin{array}{cc}-a_{11} S_{\phi} & -a_{12} S_{\phi} \\ a_{11} C_{\phi} & a_{12} C_{\phi}\end{array}\right], \quad Q(\phi)=\left[\begin{array}{cc}-b_{1} S_{\phi} & C_{\phi} \\ b_{1} C_{\phi} & S_{\phi}\end{array}\right]$.

The same is valid for each vehicle. Denoting the appropriate terms for vehicle $i$ by $q_{1 i}, \dot{q}_{1 i}, z_{i}, \tau_{i}, P_{i}, Q_{i}$ and collecting them in the vectors $q_{1}, \dot{q}_{1}, z, \tau$ and in the blockdiagonal matrices $P, Q$, respectively, then we can generalize the original method for UGVs. Hence

$$
\begin{aligned}
W \ddot{q}_{1}+\dot{W} \dot{q}_{1} & =\ddot{C} \\
W[P z+Q \tau]+\dot{W} \dot{q}_{1} & =\ddot{C} \\
\tau & =-W^{T} \lambda \\
W\left[P z-Q W^{T} \lambda\right]+\dot{W} \dot{q}_{1} & =\ddot{C} \\
W Q W^{T} \lambda & =W P z+\dot{W}_{1}-\ddot{C}
\end{aligned}
$$

from which it follows

$$
\lambda=\left(W Q W^{T}\right)^{-1}\left(W P z+\dot{W} \dot{q}_{1}-\ddot{C}\right) .
$$

The inverse of $W Q W^{T}$ exists since det $Q=-b_{1} \neq 0$ and $W$ has full row rank.

The constraint force for the $i$ th vehicle is

$$
\begin{aligned}
& \tau_{c i}=\sum_{k \in A_{c}^{i}} \sum_{j \in B_{k}}-W_{k i}^{T}\left(W_{k} Q_{i j} W_{k}^{T}\right)^{-1} \times \\
&\left(W_{k i} P_{i j} z_{i j}+\dot{W}_{k i} \dot{q}_{1, i j}+K_{d, k i} \dot{C}_{k i}+K_{p, k i} C_{k i}\right)
\end{aligned}
$$

where $A_{c}^{i}, B_{k}$ and $W_{k i}$ are defined as earlier and the environmental force is assumed to be zero. 


\subsection{Stability considerations}

The feedback loop works as follows. Each vehicle determines its state $\bar{x}_{i}=\left(x_{i}, y_{i}, \phi_{i}\right.$, $\left.\beta_{i}, r_{i}, v_{i}\right)^{T}$ and computes $\psi_{i}=\phi_{i}-\beta_{i}, z_{i}=\left(\beta_{i}, r_{i} / v_{i}\right)^{T}, P_{i}\left(\phi_{i}\right), Q_{i}\left(\phi_{i}\right), q_{1 i}=\left(x_{i}, y_{i}\right)^{T}$ and $\dot{q}_{1 i}=\left(\dot{x}_{i}, \dot{y}_{i}\right)^{T}$. The composite vectors $q_{1}, \dot{q}_{1}, z$ and the blockdiagonal matrices $P, Q$ are formed, then the constraint $C$, its Jacobian $W$ and the derivative $\dot{C}=W \dot{q}_{1}$ will be computed. For each vehicle $i$ the force $\tau_{c, i}$ will be determined using formation information and Eq. (29). Finally $\tau_{c, i}=\left(\delta_{w, i}, \alpha_{i}\right)^{T}$ will be applied as steering angle and acceleration for the vehicle. Thus the feedback loop is closed.

The constraints determine a manifold $M_{C}$. The choice of $K_{d}, K_{p}$ and $\ddot{C}+K_{d} \dot{C}+$ $K_{p} C=0$ assures that the system moves on the manifold satisfying global exponential stability (GES).

However the system of UGVs is underactuated therfore zero dynamics is present. The stability of the zero dynamics was proved in [2].

\section{Realistic dynamic modeling and control using robotic formalism}

\subsection{Geometric topology of 16 DoF ground vehicle}

Consider a tree-structured mechanical system assembled by rigid bodies $B_{j}$ for $j=$ $1, \ldots, n$, i.e. numbered from the base body to the terminals. A body can be virtual or real: virtual bodies are introduced to describe joints with multiple degrees of freedom such as ball joints or intermediate fixed frames.

Frame $K_{j}$, associated with body $B_{j}$, is given by its origin and an orthonormal basis $\left(x_{j}, y_{j}, z_{j}\right)$. Transformation between two consecutive frames $K_{i}$ and $K_{j}$ is performed by the modified Denavit-Hartenberg formalism mand can be described by the homogeneous transformation, see [11]:

${ }^{i} T_{j}=\left[\begin{array}{cc}{ }^{i} A_{j} & { }^{i} p_{j} \\ 0_{1 \times 3} & 1\end{array}\right]$

where ${ }^{i} A_{j}$ defines the $(3 \times 3)$ rotation matrix and ${ }^{i} p_{j}$ is the $(3 \times 1)$ vector describing the position of the origin of $K_{j}$ with respect to $K_{i}$. The generalized coordinate of the $j$ th joint connecting $B_{j-1}$ and $B_{j}$ is defined as follows:

$q_{j}=\bar{\sigma}_{j} \theta_{j}+\sigma_{j} r_{j}, \quad \bar{\sigma}_{j}=1-\sigma_{j}$

where $\sigma_{j}$ is 0 for rotational joints and 1 for translational joints. In the case of fixed frames attached to the same body, no joint variable is used.

The vehicle is considered as a mobile robot interconnected by joints, see Fig. 2, and modeled as a multi-body system consisting of 10 actuated and 10 virtual bodies similar to [13]. In the sequel, front steered and rear axle driven vehicle is assumed. Notice that for example $x_{4,5}$ means that the axes $x_{4}$ and $x_{5}$ are equivalent. The different joint variables are denoted by $q_{j}$. 


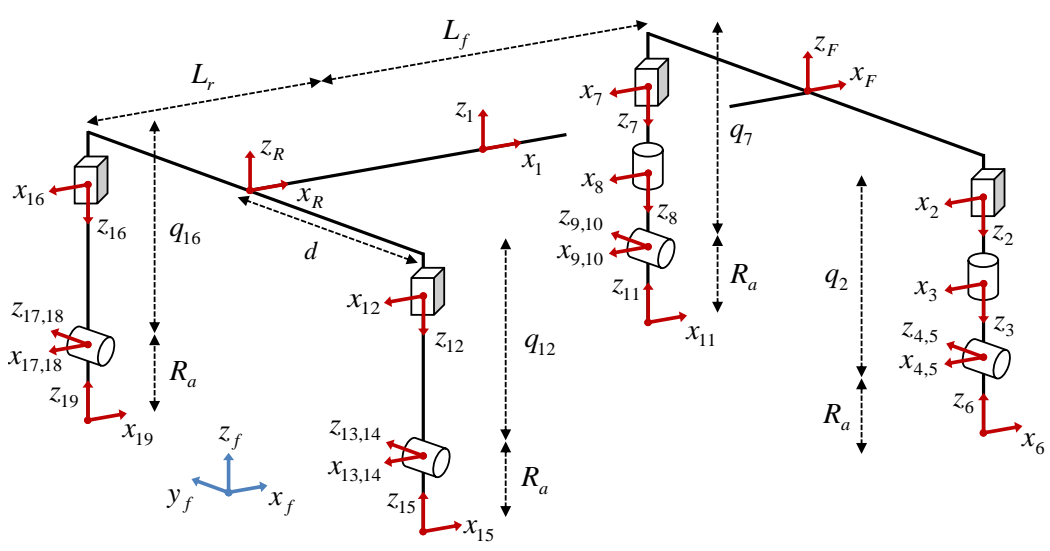

Figure 2

Model of 16 DoF ground vehicle

The 16 degree of freedom (DoF) model of the vehicle incorporates a $6 \mathrm{DoF}(x, y, z$, $\phi, \theta, \psi)$ chassis $\left(K_{1}\right)$ as the base, two front steering wheels $\left(q_{3}, q_{8}\right)$ which can be rotated about the front vertical axes, four suspensions $\left(q_{2}, q_{7}, q_{12}, q_{16}\right)$ connected to the chassis by vertical translational joints and four driving wheels $\left(q_{5}, q_{10}, q_{14}, q_{18}\right)$. Notice that for rear axle driven vehicles $\left(q_{5}, q_{10}\right)$ are not actuated.

\subsection{Novel Appell Formalism for Tree Structured Systems}

There exist several equivalent methods for mass-point systems (Newton-Euler, Lagrange, Appell) based on the common assumption that the sum of internal forces and the sum of the moment of internal forces to any point are zero which can be extended to rigid multi-body systems, see Section A.4 in [2]. Each method tends to derive the dynamic model in vector form of

$\tau=M(q) \ddot{q}+h(q, \dot{q})$

where $M(q)$ is the generalized inertia matrix and the effects of the centrifugal, Coriolis, gravity and external forces are contained in $h(q, \dot{q})$.

In our approach, Appell's method is chosen which uses the concept of acceleration energy or more precisely, the Gibbs function $[2,14]$ and eliminates a large number of numerical steps of the Newton-Euler technique. The algorithm directly computes the dynamic model of the composite system without the need of differentiation by time as in the Lagrange formalism. In case of numerical computations the complexity of the methods is different. Using symbolic computations, these differences play no more role.

The dynamic model by Appell's method reads as

$$
\frac{\partial G}{\partial \ddot{q}_{j}}+\frac{\partial P}{\partial q_{j}}=\tau_{j}
$$


where $P$ is the potential energy (gravity effect) of the segment, $q_{j}$ is the generalized variable in the direction of the generalized force $\tau_{j}$.

In order to obtain the dynamic model in the vector form $M(q) \ddot{q}+h(q, \dot{q})=\tau$ and simplify the use of Appell's method, the acceleration and angular acceleration of the segments will be computed in the compatible form

$a_{j}=\Omega_{j}(q) \ddot{q}+\theta_{j}(q, \dot{q}), \quad \varepsilon_{j}=\Gamma_{j}(q) \ddot{q}+\phi_{j}(q, \dot{q})$.

Moving from the root to the terminal of a branch in the tree structure, the matrices and vectors of the kinematic model can be computed in forward recursion. Let the antecedent of segment $j$ be $i$ and the efficient dimension of $q$ will be increased by 1 in each step, then

$$
\begin{aligned}
\Gamma_{j} & =\left[{ }^{i} A_{j}^{T} \Gamma_{i} \mid \bar{\sigma}_{j}(0,0,1)^{T}\right], \omega_{j}=\Gamma_{j} \dot{q} \\
\phi_{j} & ={ }^{i} A_{j}^{T} \phi_{i}+\bar{\sigma}_{j}\left(\omega_{y, j},-\omega_{x, j}, 0\right)^{T} \dot{q}_{j} \\
\Omega_{j} & =\left[{ }^{i} A_{j}^{T}\left(\Omega_{i}-\left[{ }^{i} p_{j} \times\right] \Gamma_{i}\right) \mid \sigma_{j}(0,0,1)^{T}\right] \\
\theta_{j} & \left.={ }^{i} A_{j}^{T}\left\{\theta_{i}+\left(\left[\phi_{i} \times\right]+\left[\omega_{i} \times\right]^{2}\right)^{i} p_{j}\right)\right\}+\sigma_{j} 2\left(\omega_{y, j},-\omega_{x, j}, 0\right)^{T} \dot{q}_{j} .
\end{aligned}
$$

After some conversions the matrix and vector portions of a single segment of the dynamic model can be written in form of

$$
\begin{aligned}
& M_{s}(q)=\left[\begin{array}{ll}
\Omega^{T} & \Gamma^{T}
\end{array}\right]\left[\begin{array}{cc}
m I_{3} & -\left[m \rho_{c} \times\right] \\
{\left[m \rho_{c} \times\right]} & J
\end{array}\right]_{s}\left[\begin{array}{c}
\Omega \\
\Gamma
\end{array}\right] \\
& h_{s}(q)=\left[\begin{array}{ll}
\Omega^{T} & \Gamma^{T}
\end{array}\right]\left[\begin{array}{c}
\theta m+\phi \times m \rho_{c}+[\omega \times][\omega \times] m \rho_{c} \\
J \phi-\theta \times m \rho_{c}+\omega \times(J \omega)
\end{array}\right]_{s}
\end{aligned}
$$

where the matrices $\Omega, \Gamma$ are already the concatenated ones, e.g. $\Omega$ is of type $3 n \times n$ and $\Gamma$ is of type $3 n \times n$.

The dynamic model (31) is the sum of the above portions if the index $s$ goes from the root to the terminals of the branches because the Gibbs function is additive. After these extensions both composite matrices $\Omega$ and $\Gamma$ have $\left(6+n_{q}\right)$ columns and $3 \cdot 21$ rows (see the number of frames in Fig. 2).

\subsection{Kinematic constraints}

Composite variables are defined to collect the parameters of the 6 DoF moving base and the generalized coordinates of the vehicle

$q_{E L}=\left[x, y, z, \varphi, \theta, \psi, q_{2}, \ldots, q_{18}\right]^{T}$.

In order to keep the vehicle in the ground, kinematic constraints are introduced that express the nullity of vertical accelerations at the contact points in the reference frame

$$
\left({ }^{f} a_{6 z},{ }^{f} a_{11 z},{ }^{f} a_{15 z},{ }^{f} a_{19 z}\right)^{T}=J_{4} \ddot{q}_{E L}+\Psi=0_{4 \times 1} .
$$


Adding the above constraint equations to the result of Eq. (31) in form of Lagrange multipliers $(\lambda)$ and assuming that no external forces act on the moving base, the direct dynamic model becomes

$$
\left(\begin{array}{c}
\ddot{q}_{E L} \\
\lambda
\end{array}\right)=\left[\begin{array}{cc}
M_{16 \times 16} & J_{4}^{T} \\
J_{4} & 0_{4 \times 4}
\end{array}\right]^{-1}\left(\begin{array}{c}
\tau-h \\
-\Psi
\end{array}\right)
$$

where $\lambda$ represent the constraint forces to maintain the contact points of the wheels on the ground and the first six components of $\tau$ are zero, see [12]. System (36) can be reformulated into a set of ordinary differential equations (ODEs) with constraints by using the well known differentiation rules $a=\dot{v}+\omega \times v$ and $\varepsilon=\dot{\omega}$ in moving frames.

\subsection{External forces}

Normal $\left(F_{z, j}\right)$, lateral $\left(F_{y, j}\right)$ and longitudinal $\left(F_{x, j}\right)$ forces at the wheel/ground contact points are taken into account in the right hand side of the dynamic model by projecting them to the base by the corresponding Jacobian matrix. Normal forces can be computed from the dynamic load distribution as described in [15] for $j=\{6,11,15,19\}$ respectively:

$$
\begin{gathered}
F_{z, j}=\frac{m}{L_{f}+L_{r}}\left(g L^{*}+\eta h^{1} a_{x}\right)\left(\frac{1}{2}+v \frac{h^{1} a_{y}}{2 g d}\right) \\
\eta=\{-1,-1,1,1\}, \quad v=\{-1,1,-1,1\}
\end{gathered}
$$

where $g$ is the gravity constant, $m$ is the total mass of the vehicle, $\eta$ and $v$ are wheel selectors and $L^{*}$ defines the static equilibrium point along the $x$-axis (front and rear are different) based on $\rho_{1 c, x}$ and $h$ is the height of the center of mass above the road surface in $K_{f}$. Since this formula does not take into consideration $\rho_{1 c, y}$ we have developed corrections for it similarly to [9].

The longitudinal and lateral wheel forces are described by Pacejka's model [16] and given by the same formula, with different coefficients, in function of the longitudinal and lateral slip

$$
\begin{aligned}
& F_{x, y}=D \sin (C \operatorname{atan}(B \alpha-E(B \alpha-\operatorname{atan}(B \alpha)))) \\
& \alpha_{x, j}=-\left({ }^{1} v_{x, j}-R_{a} \dot{q}_{j}\right) /\left(\max \left\{{ }^{1} v_{x, j}, R_{a} \dot{q}_{j}\right\}\right) \\
& \alpha_{y, j}=-\kappa q_{i}-\arctan \left(\frac{{ }^{1} v_{y, j}}{{ }^{1} v_{x, j}}\right), j=\{6,11,15,19\}
\end{aligned}
$$

where $i=\{3,8\}$ for the right and left front wheels and $\kappa$ is zero for the rear wheels. The total torques acting on the driven wheels consist of the active actuator torques and the passive longitudinal wheel forces

$\tau_{j}=\tau_{a j}-R_{a} F_{x, j}, \quad j=\{14,18\}$ 


\section{Low level decentralized control system based on re- alistic vehicle model}

\subsection{Reference signals}

Reference signals for vehicles can be derived from the acceleration pedal and the steering wheel changes, respectively. It will be assumed that they are variables for the desired longitudinal velocity and the derivative of the steering angle (whose integral is the steering angle). However, for control purposes in many cases a strategy has to be elaborated to find their time derivatives, i.e. the reference acceleration and the steering angle acceleration. Of course, if the reference signals and their derivatives are designed analytically, then this step can be omitted.

Denote with $\dot{x}_{\text {ref }}$ any variable to be differentiated further by the time and assume an approximating linear model for the output $\dot{x}$ in the form of $G_{\dot{x}, u}(s)=\frac{1}{s(1+s \tau)}$. A fictitious feedback system can be designed with PI controller $G_{u, e}(s)=k_{p}+\frac{k_{i}}{s}$, error signal $e=\dot{x}_{\text {ref }}-\dot{x}$ and open loop $G_{o}(s)=\left(k_{p} s+k_{i}\right) \frac{1}{s^{2}(1+s \tau)}$ and closed loop transfer function

$G_{\dot{x}, \dot{x}_{\mathrm{ref}}}(s)=\frac{G_{0}(s)}{1+G_{0}(s)}=\frac{k_{p} s+k_{i}}{\tau s^{3}+s^{2}+k_{p} s+k_{i}}$.

Then the derivative $\ddot{x}_{\text {ref }}$ can be approximated by the output of the controller of this fictitious closed loop system, e.g.

$\ddot{x}_{\text {ref }} \approx \frac{k_{p} s^{2}+k_{i} s}{\tau s^{3}+s^{2}+k_{p} s+k_{i}}$.

Notice, that $\tau$ is responsible for the precision of the approximation. Based on the different forms of the root locus, for high speed approximations $\tau=0.005 \mathrm{sec}$, $k_{i} / k_{p}=0.25$ and $k_{p}=100$ were chosen for the applications.

This method was applied for finding both $\dot{v}_{\text {ref }}$ and $\ddot{\delta}_{\text {ref. }}$.

\subsection{Active suspension control}

The vertical movement for passive suspensions can be taken into account as an elasticity model of

$\tau_{e j}=k_{j}\left(q_{j}-q_{j 0}\right)+F_{\mathrm{offs}, j}+d_{j} \dot{q}_{j}, \quad j=\{2,7,12,16\}$

with stiffness $k_{j}$ and damping $d_{j}$. The displacement of the suspensions is also influenced by the initial offset $F_{\text {offs, } j}$.

An important problem is the determination of the resulting total mass and center of gravity from the first moments of the chassis and the four wheel branches in steady state. Using their values and (37)-(38) the load forces can be determined. 
Then from them and the stiffness values of the suspension the vertical movements of the translational joints $q_{j 0}$ and their average value $q_{0, \text { avg }}$ can be determined. The prescribed height of $K_{1}$ above the road surface was chosen as $z_{0}=R_{a}+q_{0, \text { avg }}$ and the offsets $F_{\text {offs }, j}$ were computed to it. The passive suspension forces can be computed from them by (42).

Beside the passive suspension PID type active suspension was applied according to

$$
\tau_{j}=K_{P}\left(z_{0}-R_{a}-q_{j}\right)-K_{D} \dot{q}_{j}+K_{I} \int\left(z_{0}-R_{a}-q_{j}\right) d t,
$$

$K_{P}=100000, \quad K_{D}=10000, \quad K_{I}=300000$.

\subsection{Speed control}

For each actively driven wheel yields component-wise $\tau=\theta \ddot{q}$ where $\theta$ denotes the resulting inertia moment of the axis. The usual choice is $\dot{q}_{v, \text { ref }}=v_{\text {ref }} / R_{a}$. Hence, the PID control law

$$
\tau:=\theta\left[k_{i, v}\left(q_{v, \text { ref }}-q\right)+k_{p, v}\left(\dot{q}_{v, \text { ref }}-\dot{q}\right)+\ddot{q}_{v, \text { ref }}\right]
$$

can be suggested, from which with $e:=q_{v, \text { ref }}-q$ follows the error differential equation and from it the characteristic equation

$\ddot{e}+k_{p, v} \dot{e}+k_{i, v} e=0 \Rightarrow s^{2}+k_{p, v} s+k_{i, v}=0$.

With the choice of $(1+s T)^{2}=0$, it follows that $k_{p, v}=\frac{2}{T}$ and $k_{i, v}=\frac{1}{T^{2}}$ are satisfactory for the closed loop stability.

\subsection{Steering control}

For active steering a similar concept was chosen as for speed control, however now $\dot{q}_{D, \text { ref }}=\dot{\delta}_{w, \text { ref }}$ and its integral and derivative are $q_{D \text {,ref }}$ and $\ddot{q}_{D \text {,ref }}$, respectively. The PID control law and controller parameters were chosen similarly to active speed control.

\section{Simulation results of high level control of UGV for- mations}

Efficiency of the robust and high-speed low level control system is presented in another paper [17]. Hence, only the high level control of UGV formations is discussed here. The high level system produces reference signals for the low level system in form of acceleration $(\alpha)$ and steering angle $\left(\delta_{w}\right)$ for each vehicle of the formation. In the sequel simulation results will be presented using MATLAB/Simulink. 


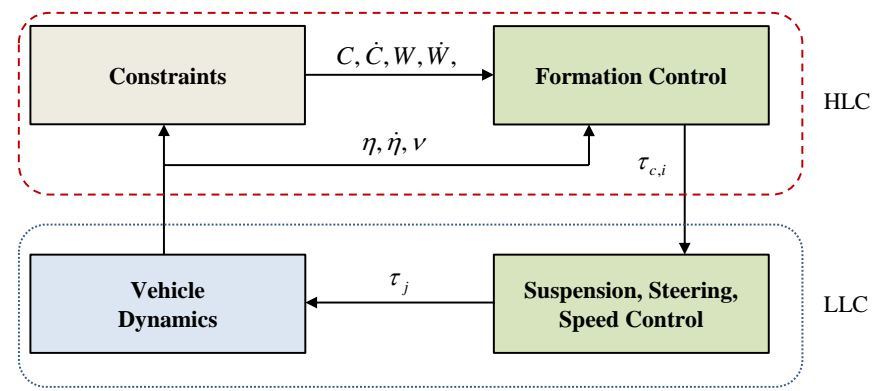

Figure 3

Structure of the hierarchical control system using multi-body interpretation

\subsection{Software system}

A software system was elaborated for the investigation of formation control of unmanned ground and marine vehicles using a broad field of methods [18]. The method based on potential function can be applied for UGVs and UMVs.

Synchronized path following was implemented only for surface ships in formation because this method is based on the dynamic model in strictly feedback form which is not valid for UGVs.

Formation control based on multi-body implementation was elaborated both for fullactuated ships and underactuated car-like vehicles. The methods allow the investigation of different types of formations, amongst horizontal, vertical, V-shaped and circular ones. The formation can be dynamically changed during the experiment.

The software has a graphical user interface in which the control method, the number of vehicles, their groups, the initial positions/orientations and the parameters of the paths, vehicles and controllers can be easily formulated. After the simulation all the states, control and other signals can be drawn and the motion of the formation is presented in animation.

From the simulation results we present here only the formation control of UGVs based on multi-body method in varying formations. For the constraints manifold $k_{d i}=k_{p i}=150$ was chosen. The structure of the control system based on multibody interpretation is shown in Fig. 3.

\subsection{Sine-shaped paths}

The master vehicle has index 1 and its orientation is $\psi_{r}=\arctan 2(A \omega \cos (\omega t), 1)$, where $A$ is the magnitude and $\omega$ is the angular velocity of the master's path. In the experiments heavy-duty cars are used therefore $A=100 \mathrm{~m}, \omega=0.02 \mathrm{rad} / \mathrm{sec}$ and $D=12.5 \mathrm{~m}$ are assumed.

In horizontal formation the reference path of the $i$-th vehicle is sin-shaped according to 


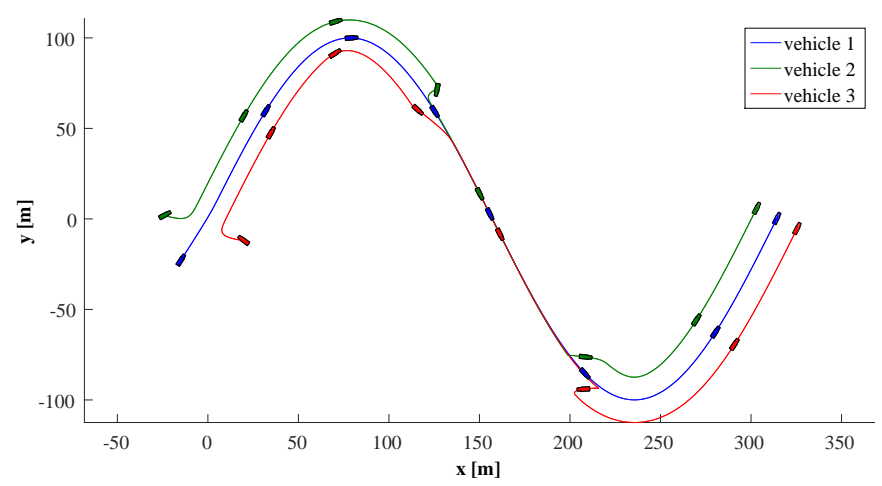

Figure 4

Realized motion of UGVs along sin-shaped path in varying formations

$$
\left(\begin{array}{c}
x_{i}(t) \\
y_{i}(t)
\end{array}\right)=\left(\begin{array}{c}
t+d_{i} \cos \left(\psi_{r}\right) \\
A \sin (\omega t)+d_{i} \sin \left(\psi_{r}\right)
\end{array}\right)
$$

where $d_{i}=(-1)^{i-1}\lfloor i / 2\rfloor D$ is the distance from the master and $D$ is the relative distance between the vehicles. Vehicles having odd index are before the master, the others are behind the master. The formation is tangential to the master's path.

Vertical formation is orthogonal to the tangent of the master's path. Vehicles having odd index are to the right from the master, while vehicles having even index are to the left from the master. The path is

$$
\left(\begin{array}{l}
x_{i}(t) \\
y_{i}(t)
\end{array}\right)=\left(\begin{array}{c}
t+d_{i} \sin \left(\psi_{r}\right) \\
A \sin (\omega t)-d_{i} \cos \left(\psi_{r}\right)
\end{array}\right)
$$

where $d_{i}$ is as for horizontal formation.

$V$-shaped formation has path

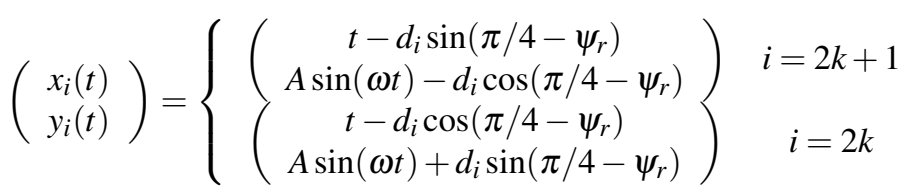

where $d_{i}=\lfloor i / 2\rfloor D$ is the distance between master and follower. The wing angle of the $\mathrm{V}$-shape is $\pi / 4$ relative to the tangent of the master's path. Vehicles having odd index are to the right from the master, the others are to the left.

Simulation results for three UGVs along sin-formed paths in varying V-shaped, horizontal and vertical formations are shown as follows. Fig. 4 presents the realized paths for the varying formations using multi-body approach. Snap-shot of the control signals along sin-formed paths are shown in Fig. 5. The snap-shot of the side slip angles along sin-formed paths can be seen in Fig. 6 illustrating that $\beta$ is small except transients belonging to larger curvatures. 

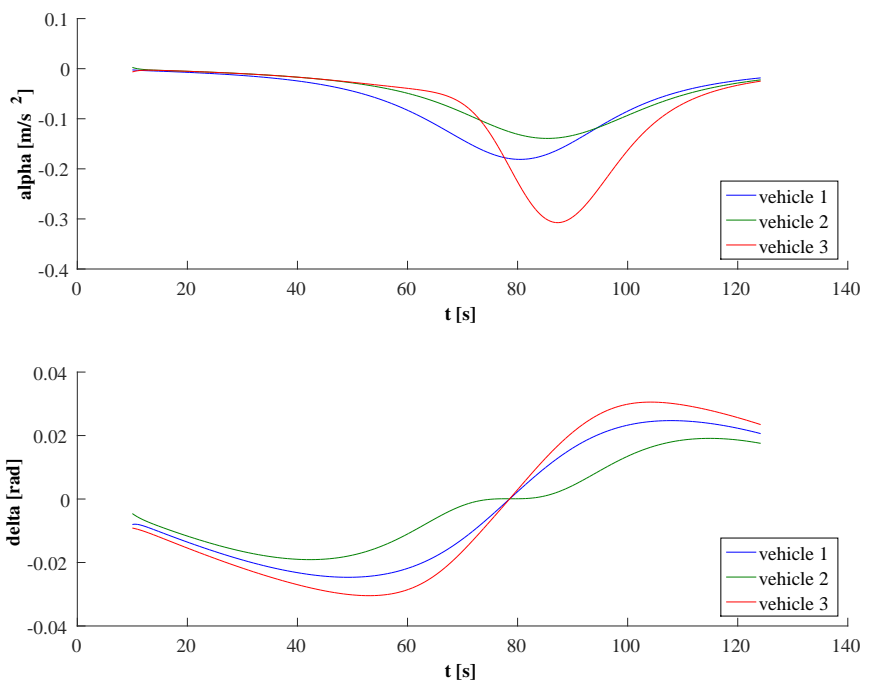

Figure 5

Snap-shot of the control signals on V-shaped section along sin-formed path

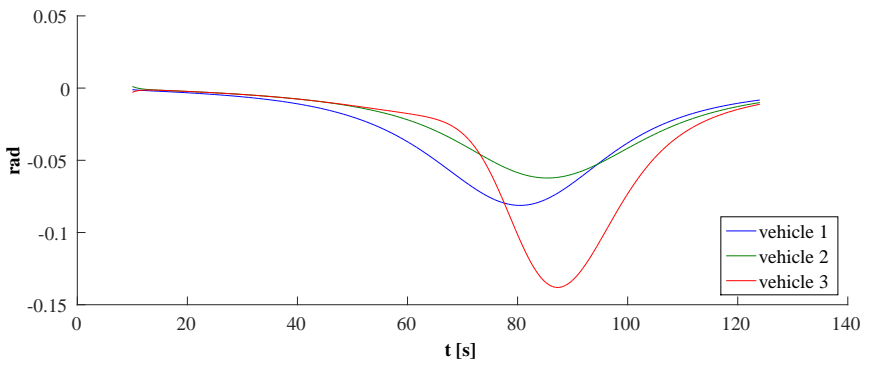

Figure 6

Snap-shot of the side-slip angles on V-shaped section along sin-formed path 


\subsection{Circular paths}

The master vehicle has index 1 and $A$ and $\omega$ are as for the sin-formed path, however the orientation is $\psi_{r}=\bmod (\omega t+\pi / 2,2 \pi / \omega)$.

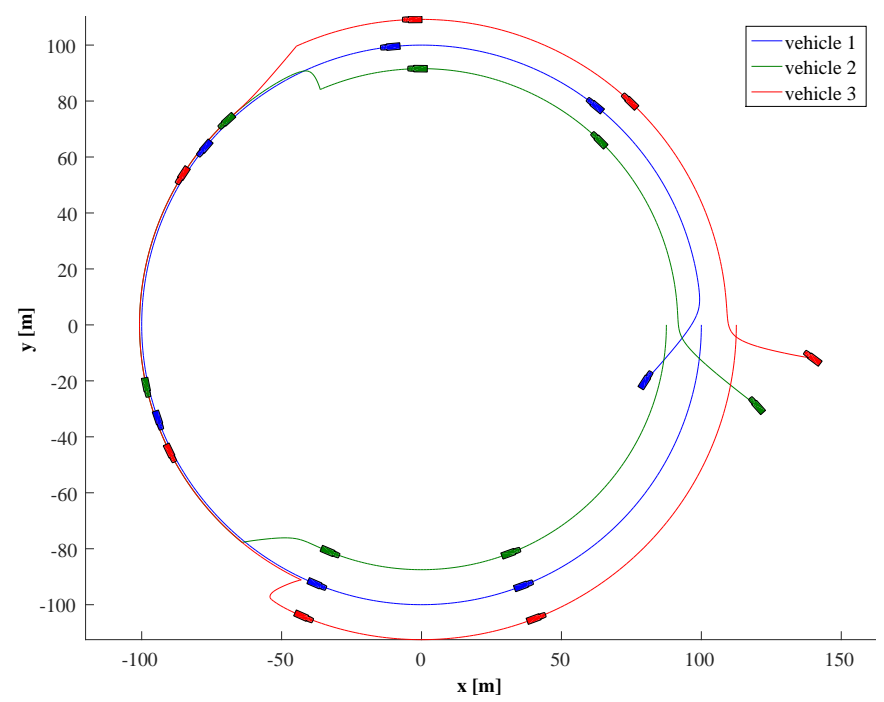

Figure 7

Realized motion of UGVs along circular paths in varying formations

In horizontal formation the circular path is defined by

$$
\left(\begin{array}{c}
x_{i}(t) \\
y_{i}(t)
\end{array}\right)=\left(\begin{array}{c}
A \cos (\omega t)+d_{i} \cos \left(\psi_{r}\right) \\
A \sin (\omega t)+d_{i} \sin \left(\psi_{r}\right)
\end{array}\right)
$$

where $d_{i}=(-1)^{i-1}\lfloor i / 2\rfloor D$ and $D$ is the relative distance between the vehicles. The formation is tangential to the master's path. Vehicles having odd index are before the master, the others are behind the master.

In vertical formation

$$
\left(\begin{array}{c}
x_{i}(t) \\
y_{i}(t)
\end{array}\right)=\left(\begin{array}{c}
A \cos (\omega t)+d_{i} \sin \left(\psi_{r}\right) \\
A \sin (\omega t)-d_{i} \cos \left(\psi_{r}\right)
\end{array}\right)
$$

where $d_{i}=(-1)^{i-1}\lfloor i / 2\rfloor D$. The formation is orthogonal to the master's path. Vehicles having odd index are to the right from the master, the others are to the left.

In $V$-shaped formation the wing angle of the $\mathrm{V}$-shape is $\pi / 4$ relative to the tangent of the master's path. Vehicles having odd index are to the right from the master, the others are to the left. The position is defined by

$$
\left(\begin{array}{c}
x_{i}(t) \\
y_{i}(t)
\end{array}\right)=\left\{\begin{array}{cc}
\left(\begin{array}{c}
A \cos (\omega t)-d_{i} \sin \left(\pi / 4-\psi_{r}\right) \\
A \sin (\omega t)-d_{i} \cos \left(\pi / 4-\psi_{r}\right)
\end{array}\right) & i=2 k+1 \\
\left(\begin{array}{c}
A \cos (\omega t)-d_{i} \cos \left(\pi / 4-\psi_{r}\right) \\
A \sin (\omega t)+d_{i} \sin \left(\pi / 4-\psi_{r}\right)
\end{array}\right) & i=2 k
\end{array}\right.
$$



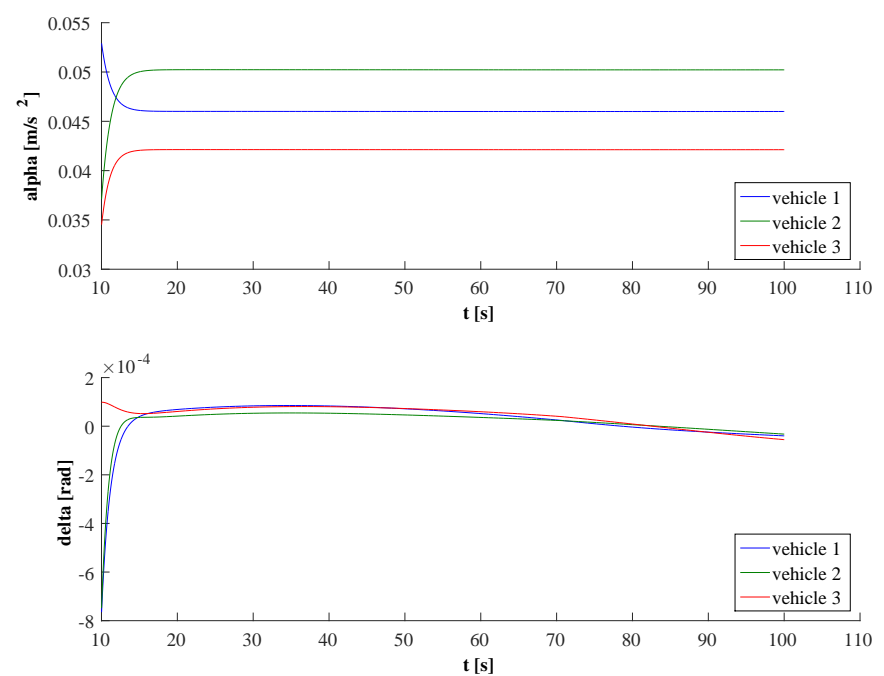

Figure 8

Snap-shot of the control signals on V-shaped section along circular path

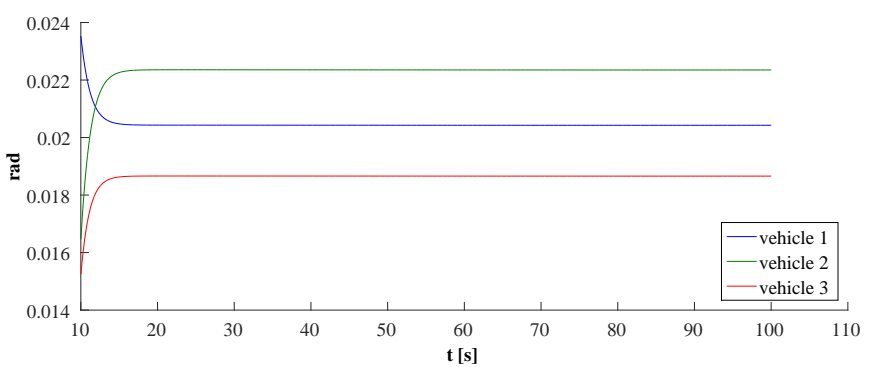

Figure 9

Snap-shot of the side-slip angles on V-shaped section along circular path

where $d_{i}=\lfloor i / 2\rfloor D$ is the distance between master and follower.

Simulation results for three UGVs along circular paths in varying V-shaped, horizontal and vertical formations are shown as follows. Fig. 7 presents the realized paths for the varying formations using multi-body approach. Snap-shot of the control signals along circular paths are shown in Fig. 8. The snap-shot of the side slip angles along circular paths can be seen in Fig. 9 illustrating that $\beta$ is small except transients belonging to larger curvatures.

\section{Conclusions}

A hierarchical control system has been elaborated for the formation control of UGVs moving in horizontal plane. The control system consists of the high level centralized formation control of the UGVs and the low level decentralized PID type suspension, 
speed and steering control of the different vehicles. Both problems were discussed in multi-body assumptions.

The formation control method, developed originally for fully-actuated ships, was generalized for underactuated car-like vehicles. Multi-body theory at this level used the result of Lanczos and the method of Lagrange multipliers. Formation specifications were formulated as constraints containing position, orientation and distance prescriptions. In order to simplify the design and implementation on formation level, approximate single track dynamic model was assumed for each vehicle.

At low level a more realistic two track dynamic model is used in the form of a multibody system in tree structure. This realistic nonlinear model is obtained by using Appell's method, Pacejka's magic formula for tyre-road connections and kinematic constraints expressing the nullity of vertical accelerations of the contact points. The interface between the higher and lower control levels is presented in the form of acceleration and steering angle prescriptions (output of high level). At low level the decentralized control system of each vehicle converts the specifications in smooth reference signals and performs the desired motion.

Simulation results of the high level control of UGV formations were presented for sine-shaped and circular paths. The Simulation results demonstrate the applicability of the multi-body approach for car-like UGVs.

Detailed simulation results for low level vehicle control based on PID type suspension, speed and steering controllers can be found in another paper [17].

Further researches are in progress to check the method under real-time conditions and state estimation based on the fusion of GPS and IMU. Further directions may be the elaboration of real-time multi-body approach for unmanned indoor quadrotor helicopters.

\section{References}

[1] T. Peni, "Feedback design methods for cooperative and constrained control problems," Ph.D. dissertation, Budapest University of Technology and Economics, 2009.

[2] B. Lantos and L. Márton, Nonlinear Control of Vehicles and Robots. Springer, 2011.

[3] I.-A. F. Ihle, M. Arcak, and T. I. Fossen, "Passivity-based designs for synchronized path-following," Automatica, vol. 43, no. 9, pp. 1508 - 1518, 2007.

[4] I.-A. F. Ihle, "Coordinated control of marine craft," Ph.D. dissertation, Norwegian University of Science and Technology, 2006.

[5] Y. Zou, P. R. Pagilla, and R. T. Ratliff, "Distributed Formation control of multiple aircraft using constraint forces," 2008, pp. 644-649. 
[6] D. Tilbury, O. Sordalen, L. Bushnell, and S. Sastry, "A multisteering trailer system: conversion into chained form using dynamic feedback," Robotics and Automation, IEEE Transactions on, vol. 11, no. 6, pp. 807-818, 1995.

[7] V. Cossalter, A. Doria, and R. Lot, "Steady turning of two-wheeled vehicles," Vehicle System Dynamics, vol. 31, no. 3, pp. 157-181, 1999.

[8] S. Maakaroun, W. Khalil, M. Gautier, and P. Chevrel, "Modeling and simulating a narrow tilting car using robotics formalism," Intelligent Transportation Systems, IEEE Transactions on, vol. 15, no. 3, pp. 1026-1038, June 2014.

[9] A. Rucco, G. Notarstefano, and J. Hauser, "Development and numerical validation of a reduced-order two-track car model," European Journal of Control, vol. 20, no. 4, pp. 163 - 171, 2014.

[10] W. Khalil, A. Vijayalingam, B. Khomutenko, I. Mukhanov, P. Lemoine, and G. Ecorchard, "OpenSYMORO: An open-source software package for symbolic modelling of robots," in Advanced Intelligent Mechatronics (AIM), 2014 IEEE/ASME International Conference on, July 2014, pp. 1206-1211.

[11] W. Khalil and J.-F. Kleinfinger, "Minimum operations and minimum parameters of the dynamic models of tree structure robots," Robotics and Automation, IEEE Journal of, vol. 3, no. 6, pp. 517-526, 1987.

[12] C. Lanczos, The Variational Principles of Mechanics. Dover Publications, 1986.

[13] S. Maakaroun, "Modélisation et simulation dynamique d'un véhicule urbain innovant en utilisant le formalisme de la robotique," Ph.D. dissertation, Ecole des Mines de Nantes, 2011.

[14] J. Somlo, B. Lantos, and P. T. Cat, Advanced Robot Control Advances in Electronics 14. Budapest, Hungary: Akademiai Kiado, 1997.

[15] U. Kiencke and L. Nielsen, Automotive Control Systems: For Engine, Driveline, and Vehicle. Springer, 2005.

[16] H. B. Pacejka, Tyre and Vehicle Dynamics, ser. Automotive engineering. Butterworth-Heinemann, 2006.

[17] G. Max and B. Lantos, "Active Suspension, Speed and Steering Control of Vehicles Using Robotic Formalism," in Proceedings of IEEE 16th International Syposium on Computational Intelligence and Informatics CINTI 2015, Budapest, Hungary, 19-21 November 2015, to appear.

[18] G. Max, "Control of Vehicles Moving in Formation,” Master's thesis, Budapest University of Technology and Economics, Hungary, 2011. 